\title{
A converse approach to the calculation of NMR shielding tensors
}

\author{
T. Thonhauser, ${ }^{1,2}$ D. Ceresoli, ${ }^{2}$ Arash A. Mostofi, ${ }^{2, *}$ Nicola Marzari, ${ }^{2}$ R. Resta, ${ }^{3}$ and David Vanderbilt ${ }^{4}$ \\ ${ }^{1}$ Department of Physics, Wake Forest University, Winston-Salem, North Carolina 27109, USA. \\ ${ }^{2}$ Department of Materials Science and Engineering, MIT, Cambridge, Massachusetts 02139, USA. \\ ${ }^{3}$ Dipartimento di Fisica Teorica Università di Trieste and INFM-DEMOCRITOS, Trieste, Italy. \\ ${ }^{4}$ Department of Physics and Astronomy, Rutgers University, Piscataway, New Jersey 08854, USA.
}

(Dated: November 13, 2018)

\begin{abstract}
We introduce an alternative approach to the first-principles calculation of NMR shielding tensors. These are obtained from the derivative of the orbital magnetization with respect to the application of a microscopic, localized magnetic dipole. The approach is simple, general, and can be applied to either isolated or periodic systems. Calculated results for simple hydrocarbons, crystalline diamond, and liquid water show very good agreement with established methods and experimental results.

PACS numbers: 71.15.-m, 71.15.Mb, 75.20.-g, 76.60.Cq
\end{abstract}

Nuclear magnetic resonance (NMR) measures the transition frequencies for the reorientation of nuclear magnetic moments in an applied magnetic field. Since the local magnetic field differs from the external one as a result of electronic screening, NMR spectroscopy [1] has been recognized since 1938 [2] to be a powerful experimental probe of local chemical environments, including structural and functional information on molecules, liquids, and increasingly, on solid-state systems.

First-principles calculations of NMR spectra were first developed in the quantum chemistry community [3] and applied to molecules and clusters, but applications to extended crystalline systems were hindered by the difficulty of including macroscopic magnetic fields, which require a non-periodic vector potential that is not compatible with Bloch symmetry. In 1996 Mauri et al. developed a linear-response approach for calculating NMR shieldings in periodic crystals based on the long-wavelength limit of a periodic modulation of the applied magnetic field [4]. In principle, this long-wavelength approach could be implemented using standard ground-state calculations, but only at the cost of introducing prohibitively large supercells. In 2001 Sebastiani and Parrinello used a localized Wannier representation [5] to derive an alternative linear-response approach based on the application of an infinitesimal uniform magnetic field [6]. More recently, attention has focused on the development of these approaches in the context of pseudopotentials [7, 8] , relying on ideas developed within the projector-augmented wave method (PAW) [9], leading to a growing use of these methods in combination with modern plane-wave pseudopotential codes [10, 11]. Despite these advances, existing methods for computing NMR shifts in crystalline systems remain complex, in that they require a linearresponse implementation with significant extra coding.

In this Letter, we reformulate the problem of computing NMR shielding tensors so that the need for a linear-response framework is circumvented. For clarity, the previous formulations shall be referred to as direct approaches, in that a magnetic field is applied and the local field at the nucleus is computed. Our alternative, converse approach obtains the NMR shifts instead from the macroscopic magnetization induced by magnetic point dipoles placed at the nuclear sites of interest. This approach is made possible in periodic systems by the recent developments that have led to the Berry-phase modern theory of magnetization [12, 13, 14, 15]. We demonstrate the method by a first application to molecules, crystals, and liquids, finding excellent agreement both with experiment and with calculations using the direct approach. Our new method is simple and general, and provides a straightforward alternative avenue to the computation of NMR shifts, which is suited for large-scale simulations and situations where a linear-response formulation is cumbersome or unfeasible.

Let us start by considering a sample to which a constant external magnetic field $\mathbf{B}^{\text {ext }}$ is applied. The field induces a current that, in turn, induces a magnetic field $\mathbf{B}^{\text {ind }}(\mathbf{r})$ such that the total magnetic field is $\mathbf{B}(\mathbf{r})=$ $\mathbf{B}^{\text {ext }}+\mathbf{B}^{\text {ind }}(\mathbf{r})$. In NMR experiments the applied fields are small compared to the typical electronic scales; the absolute chemical shielding tensor $\overleftrightarrow{\sigma}$ is then defined via the linear relationship

$$
\mathbf{B}_{s}^{\text {ind }}=-\overleftrightarrow{\sigma}_{s} \cdot \mathbf{B}^{\text {ext }}, \quad \sigma_{s, \alpha \beta}=-\frac{\partial B_{s, \alpha}^{\text {ind }}}{\partial B_{\beta}^{\text {ext }}}
$$

The index $s$ indicates that the corresponding quantity is to be taken at position $\mathbf{r}_{s}$, i.e., the site of nucleus $s$. NMR experiments usually report the isotropic shielding $\sigma_{s}=\frac{1}{3} \operatorname{Tr}\left[\overleftrightarrow{\sigma}_{s}\right]$ via a chemical shift that is defined by convention as $\delta_{s}=\sigma_{\text {ref }}-\sigma_{s}$, where $\sigma_{\text {ref }}$ is the isotropic shielding of a reference compound.

As mentioned above, direct approaches [4, 6, 7, 8] calculate the chemical shielding from the current response of the system to an external magnetic field, applied using perturbation theory and taking the long-wavelength limit. The approach we propose is fundamentally different: instead of determining the current response to a magnetic field, we derive chemical shifts from the orbital magnetization induced by a magnetic dipole. This can 
be shown using a thermodynamic relationship between mixed partial derivatives [16], as follows: Using $B_{s, \alpha}=$ $B_{\alpha}^{\text {ext }}+B_{s, \alpha}^{\text {ind }}$, Eq. (11) becomes $\delta_{\alpha \beta}-\sigma_{s, \alpha \beta}=\partial B_{s, \alpha} / \partial B_{\beta}^{\text {ext }}$. For the moment, we assume that $\mathbf{B}^{\text {ext }}$ can be replaced by the total macroscopic $B$-field in the denominator of this equation, thus neglecting the macroscopic induced field (this restriction, appropriate for normal components in a slab geometry, will be relaxed shortly). The numerator may be written as $B_{s, \alpha}=-\partial E / \partial m_{s, \alpha}$, where $E$ can be interpreted either as the energy of a virtual magnetic dipole $\mathbf{m}_{s}$ at one nuclear center $\mathbf{r}_{s}$ in the field $\mathbf{B}$ for a finite system, or as the energy per cell of a periodic lattice of such dipoles; we adopt the latter view. Then, writing the macroscopic magnetization as $M_{\beta}=-\Omega^{-1} \partial E / \partial B_{\beta}$ (where $\Omega$ is the cell volume), we obtain

$\delta_{\alpha \beta}-\sigma_{s, \alpha \beta}=-\frac{\partial}{\partial B_{\beta}} \frac{\partial E}{\partial m_{s, \alpha}}=-\frac{\partial}{\partial m_{s, \alpha}} \frac{\partial E}{\partial B_{\beta}}=\Omega \frac{\partial M_{\beta}}{\partial m_{s, \alpha}}$.

Thus, $\overleftrightarrow{\sigma}_{s}$ accounts for the shielding contribution to the macroscopic magnetization induced by a magnetic point dipole $\mathbf{m}_{s}$ sitting at nucleus $\mathbf{r}_{s}$ and all of its periodic replicas. In other words, instead of applying a constant (or long-wavelength) field $\mathbf{B}^{\text {ext }}$ to an infinite periodic system and calculating the induced field at all equivalent nuclei $s$, we apply an infinite array of magnetic dipoles to all equivalent sites $s$ and calculate the change in orbital magnetization [12, 13, 14, 15]. Since the perturbation is now periodic, it can easily be computed using finite differences of ground-state calculations. This is our principal result. Note that $\mathbf{M}=\mathbf{m}_{s} / \Omega+\mathbf{M}^{\text {ind }}$, where the first term is present merely because we have included magnetic dipoles by hand. It follows that the shielding is related to the true induced magnetization via $\sigma_{s, \alpha \beta}=-\Omega \partial M_{\beta}^{\text {ind }} / \partial m_{s, \alpha}$.

It is useful to pause here and consider the analogy with the Born [17] effective charge tensor $Z_{s, \alpha \beta}^{*}$, which may be regarded as (i) the component of the force $\mathbf{F}_{s}$ in direction $\alpha$ on site $\mathbf{r}_{s}$ by a unit macroscopic electric field $\mathcal{E}$ in direction $\beta$ (at zero nuclear displacement), or, alternatively, as (ii) the $\beta$-component of the macroscopic electric polarization $\mathbf{P}$ linearly induced by a unit displacement of nucleus $s$ and its periodic replicas in direction $\alpha$, in a vanishing macroscopic electric field. Since the force on nucleus $s$ is given by $F_{s, \alpha}=-\partial E / \partial r_{s, \alpha}$, (i) and (ii) are related by

$$
Z_{s, \alpha \beta}^{*}=-\frac{\partial}{\partial \mathcal{E}_{\beta}} \frac{\partial E}{\partial r_{s, \alpha}}=-\frac{\partial}{\partial r_{s, \alpha}} \frac{\partial E}{\partial \mathcal{E}_{\beta}}=\Omega \frac{\partial P_{\beta}}{\partial r_{s, \alpha}},
$$

in close analogy with Eq. (21). Note that, in order to comply with the Born definition, one must choose the lattice-periodical solution of Poisson's equation, corresponding to vanishing macroscopic electric field. Other choices are possible and lead to other kinds of effective charges, which are related to each other via the dielectric constant [18]. By comparing Eq. (3) to Eq. (2) we notice that the genuine analogue to $\overleftrightarrow{Z_{s}^{*}}$ is $1-\overleftrightarrow{\sigma}_{s}$ (and not $\overleftrightarrow{\sigma}_{s}$ ), as indeed the names "effective" vs. "shielding" imply. As a side note, it should be pointed out that this analogy between the electric and magnetic cases would be formally more direct if the nucleus carried a magnetic monopole charge.

As in the electrical case [18], the choice of magnetic boundary conditions implies a choice for the shape of the macroscopic finite sample. Following Ref. [19], shape effects can be embedded in the depolarization coefficients $n_{\alpha}$ (with $\sum_{\alpha} n_{\alpha}=1$ ), whose special cases are the sphere $\left(n_{x}=n_{y}=n_{z}=1 / 3\right)$, the cylinder along $z \quad\left(n_{x}=n_{y}=1 / 2\right.$, $\left.n_{z}=0\right)$, and the slab normal to $z\left(n_{x}=n_{y}=0, n_{z}=1\right)$. The main relationship for the macroscopic fields in Gaussian units may be written as $B_{\alpha}=B_{\alpha}^{\text {ext }}+4 \pi\left(1-n_{\alpha}\right) M_{\alpha}$. It can be seen that for the slab geometry the normal component of $\mathbf{B}$ coincides with the one of $\mathbf{B}^{\text {ext }}$. Hence our computed $\sigma_{s, z z}$ are suitable for direct comparison with measurements of the normal component performed on a slab-shaped sample. Assuming non-magnetic media with small, isotropic susceptibility $\chi$, it can be shown that the shielding for a general shape is related to our calculated one by $\sigma_{s, \alpha \beta}^{\text {shape }} \simeq \sigma_{s, \alpha \beta}-\delta_{\alpha \beta} 4 \pi \chi\left(1-n_{\beta}\right)$. For the special case of a spherical sample we have $\sigma_{s, \alpha \beta}^{\text {sphere }} \simeq$ $\sigma_{s, \alpha \beta}-(8 \pi / 3) \chi \delta_{\alpha \beta}$.

In order to calculate the shielding tensor of nucleus $s$ using Eq. (2), it is necessary to calculate the induced orbital magnetization due to the presence of an array of point magnetic dipoles $\mathbf{m}_{s}$ at all equivalent sites $\mathbf{r}_{s}$. The vector potential of a single dipole in Gaussian units is given by [20]

$$
\mathbf{A}_{s}(\mathbf{r})=\frac{\mathbf{m}_{s} \times\left(\mathbf{r}-\mathbf{r}_{s}\right)}{\left|\mathbf{r}-\mathbf{r}_{s}\right|^{3}} .
$$

For an array of magnetic dipoles $\mathbf{A}(\mathbf{r})=\sum_{\mathbf{R}} \mathbf{A}_{s}(\mathbf{r}-\mathbf{R})$, where $\mathbf{R}$ is a lattice vector. Since $\mathbf{A}$ is periodic, the average of its magnetic field $\nabla \times \mathbf{A}$ over the unit cell vanishes; thus, the eigenstates of the Hamiltonian remain Bloch-representable. In the Fourier representation $\mathbf{A}(\mathbf{r})=\sum_{\mathbf{G} \neq 0} \tilde{\mathbf{A}}(\mathbf{G}) \mathrm{e}^{i \mathbf{G} \cdot \mathbf{r}}$ with

$$
\tilde{\mathbf{A}}(\mathbf{G})=-\frac{4 \pi i}{\Omega} \frac{\mathbf{m}_{s} \times \mathbf{G}}{G^{2}} \mathrm{e}^{-i \mathbf{G} \cdot \mathbf{r}_{s}},
$$

where the reciprocal lattice vector $\mathbf{G}=0$ may be excluded from the sum with no loss of generality. Note that we have implicitly chosen the transverse gauge $\nabla \cdot \mathbf{A}=0$, which is apparent from $\mathbf{G} \cdot\left(\mathbf{m}_{s} \times \mathbf{G}\right)=0$. The periodic vector potential $\mathbf{A}(\mathbf{r})$ can now be included in the Hamiltonian with the usual substitution for the momentum operator $\mathbf{p} \rightarrow \mathbf{p}-\frac{e}{c} \mathbf{A}$. As a result, the kinetic energy operator becomes

$$
\frac{p^{2}}{2 m_{e}} \longrightarrow \frac{p^{2}}{2 m_{e}}-\frac{e}{m_{e} c} \mathbf{A} \cdot \mathbf{p}+\frac{e^{2}}{2 m_{e} c^{2}} A^{2},
$$

where $m_{e}$ is the electronic mass and $c$ is the speed of light. Due to our choice of gauge, $\mathbf{p}$ and $\mathbf{A}$ commute. 
We can now calculate the shielding according to Eq. (2) by solving for the ground state with the additional terms of Eq. (6) included in the Hamiltonian 21, and then calculating the resulting change in orbital magnetization.

The converse method can be implemented directly in any all-electron electronic-structure code. However, many popular density-functional theory codes use pseudopotentials to increase computational efficiency. In order to calculate NMR shifts in the presence of pseudopotentials, a PAW reconstruction [9] needs to be performed, as shown by Pickard and Mauri [7]. We have developed this reconstruction methodology for the converse method; the rather involved mathematical formalism will be presented elsewhere [22]. We implemented our converse approach, including this reconstruction and the calculation of orbital magnetization [12, 13, 14, 15], into the PWscF package of the QUANTUM-ESPRESSO distribution 23]. We use the PBE exchange-correlation functional [24] and Troullier-Martins pseudopotentials [25], with convergence of the NMR shifts for a kinetic-energy cutoff of 80 Ryd. The dipole perturbation $\left|\mathbf{m}_{s}\right|$ used is $1 \mu_{\mathrm{B}}$, although we find identical results for any value in the broad range $10^{-6}$ to $10^{3} \mu_{\mathrm{B}}$.

As an initial test, we applied our converse approach to the calculation of hydrogen NMR chemical shieldings for small molecules using a supercell geometry (in such cases the full orbital-magnetization theory is not needed, as the magnetization can be obtained just by integrating the orbital currents over the molecular region, but the results presented here were in fact obtained using the Berry-phase modern theory of magnetization). For purposes of comparison, we also calculated these shieldings with the direct method, also implemented by some of us in PWSCF, largely eliminating discrepancies due to any technicality. The results for these two approaches are shown in Table @ together with the experimental values. It is immediately obvious that the direct and converse methods give almost identical results, validating our approach, and also very good agreement (we suspect that the slight deviations between the two calculations are due to the long-wavelength approximation of the direct method).

Next, we applied our method to crystalline diamond. For our calculations we used an 8-atom cubic cell with a lattice constant of $3.498 \AA$. The NMR shielding converged to within $0.1 \mathrm{ppm}$ for a k-point mesh of $8 \times 8 \times 8$. For the ${ }^{13} \mathrm{C}$ shielding we find $131.20 \mathrm{ppm}$, in perfect agreement with the direct method. To estimate the effect of the spurious interactions of the localized dipole with its images in neighboring supercells, we repeated the calculation for a 64-atom cubic cell, finding an almost identical shielding of $131.24 \mathrm{ppm}$. The fast convergence with respect to supercell size is due to the fast decay $\left(1 / r^{2}\right)$ of the vector potential in real space.

Finally, we applied the converse approach to compute the hydrogen chemical shifts in a supercell simu-
TABLE I: Hydrogen NMR chemical shielding $\sigma$, in ppm, for several different molecules. Structural parameters were taken from footnote 22 of Ref. [4].

\begin{tabular}{lccr}
\hline \hline & experiment & direct & converse \\
\hline $\mathrm{H}_{2}$ & $26.26^{a}$ & 26.2 & 26.2 \\
$\mathrm{HF}$ & $28.51^{a}$ & 28.4 & 28.5 \\
$\mathrm{CH}_{4}$ & $30.61^{a}$ & 30.8 & 31.0 \\
$\mathrm{C}_{2} \mathrm{H}_{2}$ & $29.26^{b}$ & 28.8 & 28.9 \\
$\mathrm{C}_{2} \mathrm{H}_{4}$ & $25.43^{b}$ & 24.7 & 24.8 \\
$\mathrm{C}_{2} \mathrm{H}_{6}$ & $29.86^{b}$ & 30.2 & 30.4 \\
\hline \hline
\end{tabular}

${ }^{a}$ Reference [26].

${ }^{b}$ Reference [27].

lation of liquid water. Our supercell contained 64 water molecules, twice the size of the largest supercell used in previous NMR calculations on liquid water using the direct method [28, 29]. We obtained the atomic trajectories from a molecular-dynamics simulation using TIP4P [30] potentials under standard conditions. For five snapshots separated by 200 ps, we took the atomic positions and thermalized the hydrogen atoms alone for 2 ps using ab-initio Car-Parrinello molecular dynamics. This procedure is aimed at obtaining a more realistic description of the detailed structure of the water molecules while retaining the accuracy of the oxygen-oxygen pair correlation function. We then used these positions to calculate hydrogen shifts with the converse method. We calculated the shift of liquid water with reference to the gas-phase shift, i.e., $\delta_{\text {liquid }}=\sigma_{\text {gas }}-\sigma_{\text {liquid, }}$ thus reporting the experimental measurable change for the gasliquid transition. For the gas-phase shift the converse method gives 31.0 ppm. For the susceptibility correction of periodic water we used the experimental value for $\chi_{\text {water }}=-7.2 \times 10^{-7}$ emu under standard conditions 31]. Our distribution for the hydrogen shifts, shown in Fig. 1. can be directly compared to results obtained using the direct method reported in Fig. 4 of Ref. 28] and in Ref. 29]. We find an average shift of $5.94 \mathrm{ppm}$ from our distribution, in excellent agreement with $5.83 \mathrm{ppm}$ and 5.15 ppm from Refs. [28] and [29], respectively. Furthermore, the spread of our distribution as measured by the standard deviation is $2.4 \mathrm{ppm}$, again in precise agreement with the value of $2.4 \mathrm{ppm}$ obtained from the direct method [29].

At first sight it might appear that the converse method is computationally more demanding than the direct method, since we need to perform $3 N$ calculations to obtain the shielding tensor for $N$ atoms. Often, though, only a few selected shifts are needed, and even in the worst-case scenario (such as the water calculation shown before) it should be stressed that re-minimizing the electronic wavefunctions in the presence of the perturbation is very fast, usually requiring a single self-consistent iteration. This enables the calculation of NMR shielding 


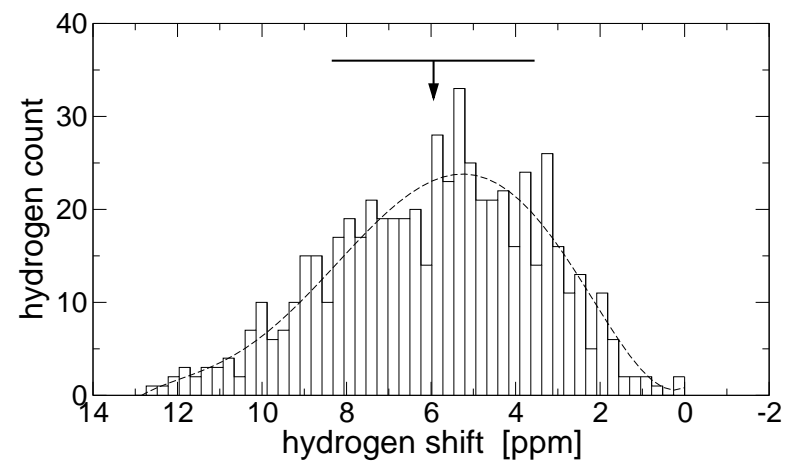

FIG. 1: Distribution of hydrogen NMR shifts in liquid water relative to the gas-phase shift. The distribution was obtained from five snapshots of the 64-molecule system (640 hydrogen atoms). The dashed line is a polynomial fit and it serves as a guide to the eye. The vertical arrow and horizontal line indicate the position of the average and the range of the standard deviation, respectively.

tensors for systems with several hundred atoms even on small computer clusters. However, the main advantage of the converse method is the simplicity of its implementation, in that it works via finite differences of ground-state calculations and does not require a linear-response implementation. This is likely to be a significant advantage for future applications in conjunction with more complex forms of exchange-correlation functionals such as $\mathrm{DFT}+\mathrm{U}$, exact exchange, hybrid functionals, or beyondDFT correlated-electrons methods.

In conclusion, we have derived an alternative firstprinciples method for calculating NMR chemical shielding tensors in solids. The new approach is considerably simpler than existing techniques, avoiding altogether the difficulties related to the choice of a gauge origin and the need for a linear-response implementation. We have demonstrated the correctness and viability of our approach by calculating chemical shieldings in isolated and periodic systems: simple molecules, crystalline diamond, and liquid water, finding excellent agreement with previous theoretical and experimental results. Applications to more complex systems, including small proteins and systems with heavy elements, are currently in progress.

This work was supported by NSF grant DMR-0549198, ONR grant N00014-07-1-1095, the DOE/SciDAC project on Quantum Simulation of Materials and Nanostructures, and MITEI/ENI. All computations were performed on the WFU DEAC Cluster with support from the WFU Science Research Fund.

* Current address: Department of Materials, Imperial College London, SW7 2AZ, United Kingdom.

[1] Encyclopedia of NMR, edited by D.M. Grant and R.K. Harris (Wiley, London, 1996).
[2] I. I. Rabi, J. R. Zacharias, S. Millman, and P. Kusch, Phys. Rev. 53, 318 (1938).

[3] W. Kutzelnigg, U. Fleischer, and M. Schindler, NMR Basic Principles and Progress (Springer, Berlin, 1990).

[4] F. Mauri, B. G. Pfrommer, and S. G. Louie, Phys. Rev. Lett. 77, 5300 (1996).

[5] N. Marzari and D. Vanderbilt, Phys. Rev. B 56, 12847 (1997).

[6] D. Sebastiani and M. Parrinello, J. Phys. Chem. A 105, 1951 (2001).

[7] C. J. Pickard and F. Mauri, Phys. Rev. B 63, 245101 (2001); Phys. Rev. Lett. 91, 196401 (2003).

[8] J. R. Yates, C. J. Pickard, and F. Mauri, Phys. Rev. B 76, 024401 (2007).

[9] P. E. Blöchl, Phys. Rev. B 50, 17953 (1994).

[10] B. G. Pfrommer, J. Demmel, and H. Simon, J. Comp. Phys. 150, 287 (1999); B. G. Pfrommer, M. Coté, S. G. Louie, and M. L. Cohen, J. Comp. Phys. 131, 233 (1997).

[11] S. J. Clark, M. D. Segall, C. J. Pickard, P. J. Hasnip, M. J. Probert, K. Refson, M. C. Payne, Zeitschrift für Kristallographie 220, 567 (2005).

[12] R. Resta, D. Ceresoli, T. Thonhauser, and David Vanderbilt, ChemPhysChem 6, 1815 (2005).

[13] T. Thonhauser, D. Ceresoli, D. Vanderbilt, and R. Resta, Phys. Rev. Lett. 95, 137205 (2005).

[14] D. Ceresoli, T. Thonhauser, D. Vanderbilt, and R. Resta, Phys. Rev. B 74, 24408 (2006).

[15] Di Xiao, J. Shi, and Q. Niu, Phys. Rev. Lett. 95, 137204 (2005); J. Shi, G. Vignale, Di Xiao, and Q. Niu, Phys. Rev. Lett. 99, 197202 (2007).

[16] R. Ditchfield, J. Chem. Phys. 56, 5688 (1972); W. Kutzelnigg, Isr. J. Chem. 19, 193 (1980).

[17] R. Resta, Rev. Mod. Phys. 66, 899 (1994).

[18] Ph. Ghosez, J.-P. Michenaud, and X. Gonze, Phys. Rev. B 58, 6224 (1998).

[19] L. D. Landau and E. M. Lifshitz, Electrodynamics of Continuous Media (Pergamon Press, Oxford, 1960).

[20] J. D. Jackson, Classical Electrodynamics, $2^{\text {nd }} e d$. (Wiley, New York, 1975).

[21] Insofar as our goal is to extrapolate the finite-difference results to reproduce the linearly-induced magnetization, the $A^{2}$ term has no influence and may be neglected.

[22] D. Ceresoli, N. Marzari, G. Lopez, and T. Thonhauser, in preparation.

[23] P. Giannozzi et al., http://www.quantum-espresso.org.

[24] J. P. Perdew, K. Burke, and M. Ernzerhof, Phys. Rev. Lett. 78, 1396 (1997).

[25] N. Troullier and J. L. Martins, Phys. Rev. B 43, 1993 (1991).

[26] W. T. Raynes, Nuclear Magnetic Resonance (The Chemical Society, London, 1977), Vol. 7.

[27] Values of $\sigma$ relative to $\mathrm{CH}_{4}$ are reported in W. G. Schneider, H. J. Bernstein, and J. A. Pople, J. Chem. Phys. 28, 601 (1958). Reference [26] can then be used to convert to the absolute scale.

[28] D. Sebastiani and M. Parrinello, ChemPhysChem 3, 675 (2002).

[29] B. G. Pformer, F. Mauri, and S. G. Louie, J. Am. Chem. Soc. 122, 123 (2000).

[30] W. L. Jorgensen, J. Chandrasekhar, and J. D. Madura, J. Chem. Phys. 79, 926 (1983).

[31] David R. Lide, ed., CRC Handbook of Chemistry and Physics, 71th Edition (CRC Press, Boston). 